\title{
Extending the analysis of the dilution method to obtain the phytoplankton concentration at which microzooplankton grazing becomes saturated
}

\author{
Anna M. Redden ${ }^{1, *}$, Brian G. Sanderson ${ }^{2}$, David Rissik ${ }^{3}$ \\ ${ }^{1}$ School of Applied Sciences, University of Newcastle, PO Box 127 Ourimbah, NSW 2250, Australia \\ ${ }^{2}$ Environmental Modelling Solutions, 38 Dora St, NSW 2250, Australia \\ ${ }^{3}$ Estuaries Branch, Ecosystems Directorate, Dept Land and Water Conservation, GPO Box 39, Sydney, NSW 2001, Australia
}

\begin{abstract}
The application of the dilution method to productive coastal waters has been limited, in part by difficulties in interpreting the nonlinear grazing dynamics. A split-function model of microzooplankton grazing is used to adapt the dilution method for analysis of mesotrophic and eutrophic systems. Phytoplankton growth rate $\mu$ and microzooplankton grazing rate $g$ are obtained using the conventional analysis with dilutions sufficient to ensure that grazing rate is proportional to phytoplankton concentration. When phytoplankton concentration is sufficiently great, dilution experiments indicate that grazing becomes independent of phytoplankton concentration (i.e. grazing is saturated). A straightforward calculation yields the phytoplankton concentration $P_{\mathrm{s}}$ that saturates grazing. The saturated grazing is $G_{\mathrm{s}}=g P_{\mathrm{s}}$. Grazing balances primary production when the phytoplankton concentration is $P_{\mathrm{b}}=g P_{\mathrm{s}} / \mu$. In productive waters, determination of $P_{\mathrm{s}}$ is important because microzooplankton grazing becomes an increasingly irrelevant constraint on exponential phytoplankton growth as the phytoplankton concentration further exceeds $P_{\mathrm{b}}$. Data were analyzed from a dilution experiment conducted in a mesotrophic coastal lagoon in New South Wales, Australia. Values of

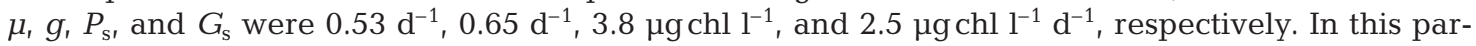
ticular experiment the in situ phytoplankton concentration exceeded $P_{\mathrm{b}}$ by a factor of 1.7.
\end{abstract}

KEY WORDS: Microzooplankton grazing • Dilution method • Nonlinear models • Zooplankton • Phytoplankton growth · Saturated grazing

\section{INTRODUCTION}

The dilution method, developed by Landry \& Hassett (1982), has been widely applied to simultaneously estimate rates of phytoplankton growth and microzooplankton grazing (e.g. Landry 1994, Gifford et al. 1995, Putland 2000, and references therein). In the dilution method, a large sample of whole water containing phytoplankton and grazers is collected and used to prepare a series of diluted samples using various amounts of filtered water. It is assumed that diluting does not modify the growth rate of phytoplankton but does reduce the grazing rate because the concentra-

*E-mail: ctamr@cc.newcastle.edu.au tion of zooplankton grazers is reduced. The dilution series is then incubated, usually for a diurnal cycle under natural light. The concentration of chlorophyll a in diluted samples is measured before and after incubation, from which an apparent growth rate can be obtained for the phytoplankton. Larger apparent phytoplankton growth rates are usually obtained from the more dilute samples, because the grazing rate is reduced by dilution whereas phytoplankton growth rate is independent of dilution. Grazing rate and phytoplankton growth rate can be estimated by using an appropriate mathematical model to analyze measurements from a dilution experiment. The method was originally applied using a linear model, appropriate for typical oligotrophic waters, in which phytoplankton growth rate $\mu$ is independent of phytoplankton con- 
centration, whereas grazing rate $g$ is proportional to zooplankton concentration (Landry \& Hassett 1982).

Data from dilution experiments can also be analyzed in the context of nonlinear grazing models, which are likely to apply in mesotrophic and eutrophic systems. Gallegos (1989) fitted a variety of nonlinear models to data from a dilution experiment conducted in a eutrophic system. In general, nonlinear models do not have a simple interpretation and are analytically intractable. Here, we will analyze a particularly simple nonlinear model that fits the data, is analytically tractable, and provides fundamental measures that are easy to interpret. These measures include phytoplankton growth rate, microzooplankton grazing rate, and the phytoplankton concentration at which grazing becomes saturated.

When fitting nontrivial mathematical models to data there are 3 types of error that may or may not arise. First, there are errors inherent to all experimental measurements. Second, the mathematical technique used to solve or fit the equations to the data may not be exact. Third, there is the possibility that we may be fitting the wrong equations (model) to the data.

Gallegos (1989) used approximate mathematical techniques to fit dilution data to nonlinear models, whereby grazing rate was a function of dilution. The mathematical technique used to analyze data in Gallegos (1989) had 2 potential deficiencies belonging to the second category above. First, the '3-point' method implicit in Eq. (8) of Gallegos (1989) uses an extrapolation and is, therefore, sensitive to experimental error and natural biological variability. Second, Gallegos (1989) uses the approximation that 'alterations of clearance rates by the microzooplankton community is a response to the initial dilution treatment, but clearance rates otherwise remain temporally constant within all dilutions'. In other words, Gallegos assumes that microzooplankton grazing does not respond to variation in phytoplankton concentration during incubation. This second approximation appears to be made for the sake of mathematical convenience because Gallegos (1989) inserts a nonlinear grazing dynamic into the solution for the differential equation for linear growth and linear grazing dynamics. Such mathematical approximations are reasonable for small perturbations about the initial state.

Early theoretical work (Holling 1959) suggests that grazing dynamics might exhibit the following characteristics. When phytoplankton concentration is below some threshold then grazing ceases; when it is above some saturation level, then grazing is constant. At intermediate phytoplankton concentrations, grazing increases in proportion to the concentration of phytoplankton. This might be represented as a piecewise linear model. Alternatively, the 3 behaviors might be considered as asymptotic limits on some general nonlinear function. Gallegos (1989) considered a general nonlinear function and was therefore constrained to using an approximate mathematical treatment in order to fit data from dilution experiments to theory. This is surprising since the dilution series plotted in Fig. 3 of Gallegos (1989) is particularly well suited to a piecewise linear model with nonlinearity manifesting as a saturated feeding response.

Evans \& Paranjape (1992) analyze the fit of analytically intractable, nonlinear grazing models to data from dilution experiments, as well as synthetic data. Specifically, grazing functions with the forms of either a MichaelisMenton hyperbola or a hyperbolic tangent were considered. Like Gallegos, they use numerical methods to achieve a fit. Importantly, Evans \& Paranjape (1992) quantify the numerical error of their calculation and show it to be unimportant. They also show that neither of their nonlinear grazing models can be rejected as being inconsistent with their measurements, but the linear grazing model cannot be rejected either.

Evans \& Paranjape (1992) chose not to accept the simplest model that cannot be shown to be inconsistent with the data. This position is justified, in part, by the widely held notion that a nonlinear grazing response is a real possibility and therefore deserves to be analyzed in its own right. Evans \& Paranjape (1992) used their analysis to demonstrate some aspects of the uncertainty in estimates of phytoplankton growth rates and zooplankton grazing rates. Interestingly, they did not consider a piecewise linear grazing model, which would also fit their observations at least as well as the other nonlinear grazing functions they considered.

Landry et al. (1995) use an extended experimental protocol to obtain phytoplankton growth and community microzooplankton grazing rates. The extended technique recognizes that dilution is a proxy variable for the relative microzooplankton grazing. Application of the modified experimental methodology to oligotrophic communities confirmed a one-to-one linear relationship between dilution and the relative microzooplankton grazing obtained using tracer concentrations of fluorescently labeled bacteria. The application of this extended method, or some variant, to mesotrophic systems remains to be demonstrated. A mesotrophic experiment would be useful because it could provide a direct measurement of the form of the nonlinear grazing function.

Dolan et al. (2000) find that growth of some microzooplankton groups can be substantial when undiluted and slightly diluted samples are incubated. They also demonstrate that grazer mortality can occur in highly diluted treatments. Most importantly, Dolan et al. (2000) show that these effects can be quantified by augmenting dilution experiments with an examination of the abundances of different groups of microzooplankters. The usefulness of the dilution method is thereby 
extended to determine features of zooplankton growth and community structure, as well as grazing.

It is interesting to note that for both of the Dolan et al. (2000) experiments the apparent phytoplankton growth is independent of dilution when the fraction of unfiltered seawater is 0.8 or greater. Indeed, Dolan et al. (2000) fit both linear and nonlinear models to their second experiment, in which apparent growth seems independent of dilution for fractions of unfiltered seawater with values $0.4,0.8$ and 1.0 .

The following work presents data that obviously cannot be fitted using a linear grazing model, but is clearly compatible with a piecewise linear grazing model. Piecewise linear grazing provides the most straightforward model that could be deemed compatible with our measurements, as well as those plotted in Fig. 3 of Gallegos (1989). Analytic solutions of the piecewise linear model are developed and shown to be easily interpreted and readily applied to the analysis of dilution experiments.

Errors of the second type can be avoided by appropriate use of analytic solutions. It is possible to numerically or otherwise approximately fit the piecewise linear model to data (Gallegos 1989), but this can result in lost insight and destroys the simplicity of analysis.

We describe a dilution experiment conducted using a $40 \mathrm{l}$ whole water sample from Dee Why Lagoon in New South Wales on the east coast of Australia. In the experiment reported here, the prescreening step to remove zooplankton $>200 \mu \mathrm{m}$ was not conducted. Larger zooplankters were retained to maintain near natural conditions and to help stabilize microzooplankton abundance. Data from this dilution experiment indicate nonlinear zooplankton grazing that also appears to be well represented using a piecewise linear model to treat the saturated zooplankton grazing. There is no evidence of grazing ceasing at low phytoplankton concentrations. Thus the system can be described using 3 constants: the growth rate of phytoplankton $\mu\left(\mathrm{d}^{-1}\right)$, the grazing rate $g\left(\mathrm{~d}^{-1}\right)$, and the phytoplankton concentration $P_{\mathrm{s}}\left(\mu \mathrm{g} \mathrm{chl} \mathrm{l}^{-1}\right)$ at which the grazing becomes saturated. Using Eqs (3) \& (6) below, it is easy to determine these three constants without changing the experimental protocol. The saturated grazing is then given by $G_{\mathrm{s}}=g P_{\mathrm{s}}$. In the interests of clarity and completeness, the treatment below will be developed from first principles.

\section{PHYTOPLANKTON GROWTH AND LINEAR MICROZOOPLANKTON GRAZING}

The equation for the instantaneous rate of change of a natural phytoplankton concentration $P$ in the presence of zooplankton at some concentration $Z$ is often written:

$$
\frac{\mathrm{d} P}{\mathrm{~d} t}=\mu P-\gamma Z P
$$

where $\mu$ is the growth rate of phytoplankton in the absence of grazing by zooplankton and $\gamma$ is the grazing rate per unit zooplankton concentration. The instantaneous rate of loss of phytoplankton due to zooplankton grazing is commonly just called grazing. Grazing is proportional to the zooplankton concentration $Z$ multiplied by the phytoplankton concentration $P$ as represented by the rightmost term $\gamma Z P$ in Eq. (1). In the absence of other constraints, the phytoplankton will grow exponentially or decay exponentially depending upon whether the grazing rate $g=\gamma Z$ is less than or greater than $\mu$.

\section{REVIEW OF THE DILUTION METHOD FOR MEASURING $g$ AND $\mu$}

Consider some undiluted sample with an initial phytoplankton concentration $P_{0}$ and an unchanging zooplankton concentration $Z$. If the sample is diluted with filtered seawater so the fraction of unfiltered seawater is $0<D \leq 1$, then the equation describing the rate of change of the diluted phytoplankton concentration $p$ is:

$$
\frac{\mathrm{d} p}{\mathrm{~d} t}=\mu p-\gamma D Z p=\mu p-g D p
$$

The biological processes represented in Eq. (2) are identical to those represented in Eq. (1); all that has changed are the concentrations.

In Eq. (2) the diluted sample has a zooplankton concentration $D Z$, which is considered not to change over the incubation period. Provided that microzooplankton grow slowly compared to phytoplankton, it is appropriate to approximate $Z$ as being constant over sufficiently short incubation periods. A $1 \mathrm{~d}$ incubation is typically used in order to obtain values for $g$ and $\mu$ that are averaged over the daily cycle of irradiance. Landry (1994) and Gallegos et al. (1996) discuss modifications to the dilution technique when $Z$ changes during the incubation. Dolan et al. (2000) further consider the ramifications of zooplankton growth in relatively undiluted samples and also the mortality of some groups of grazers in more dilute samples.

The initial value of the diluted phytoplankton concentration is $p_{0}=P_{0} D$. The grazing rate in the natural system (undiluted sample) is $g=\gamma Z$. In the diluted sample the grazing rate is $g D$, which is less than that in the undiluted sample because the concentration of grazers is reduced.

The solution to Eq. (2) can be obtained using separation of variables and can be arranged into the following 
expression for the apparent growth rate:

$$
\frac{1}{t} \ln \frac{p(t)}{p_{0}}=\mu-g D
$$

which trivially yields $\mu$ and $g$ from measurements of $p(t)$ over some incubation period $t$ using samples with several different fractions of unfiltered seawater $D$.

Fig. 1 shows data from a dilution experiment plotted according to Eq. (3). A linear line with negative slope fits experimental results from greatly diluted samples $(D<0.3)$. This linear fit yields the phytoplankton growth rate $\mu=0.53 \mathrm{~d}^{-1}$, and the grazing rate $g=0.65 \mathrm{~d}^{-1}$. At high concentrations $(D \geq 0.5)$ the apparent growth appears to become independent of the fraction of unfiltered seawater. This might be attributed to the concentration of $p$ being so high that grazing is not limited by food availability - a circumstance subsequently referred to as saturated grazing.

\section{THE DILUTION METHOD FOR MEASURING SATURATED GRAZING}

At sufficiently low phytoplankton concentrations it is reasonable to assume that grazing is limited by the rate at which zooplankton can capture phytoplankton. The mass of phytoplankton grazed per unit time will, there-

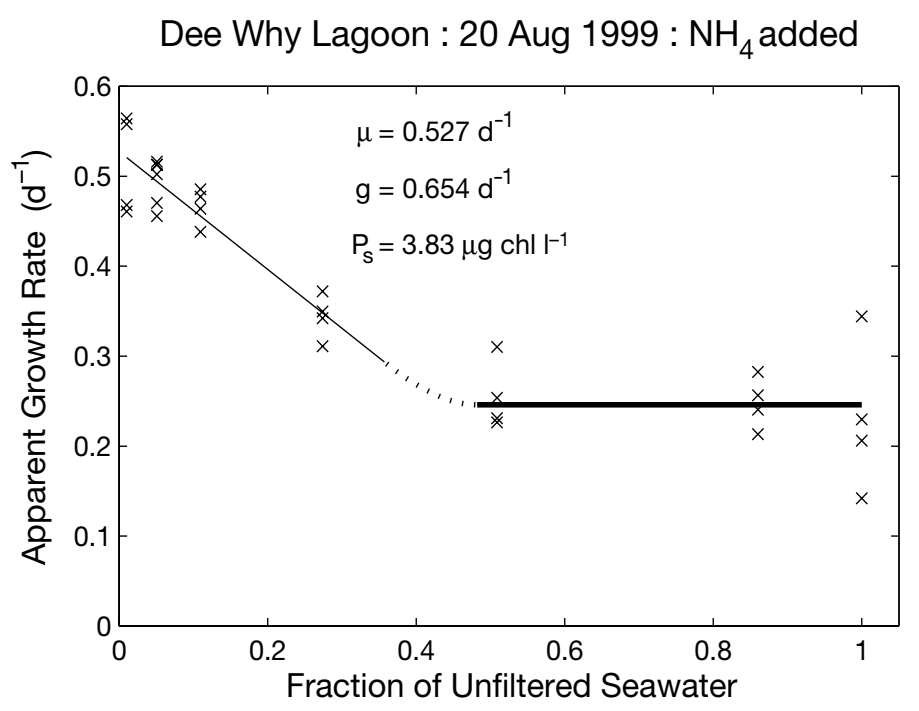

Fig. 1. Apparent growth rate, $(1 / t) \ln \left[p(t) / p_{0}\right]$ plotted against fraction unfiltered seawater $D=p_{0} / P_{0}$. Crosses show values from a dilution experiment. The thin line shows the best fit using linear grazing when $p_{0}$ and $p(t)$ are both less than $P_{\mathrm{s}}$. The $y$-intercept is $\mu$ and the slope is $g$. The thick line shows the best fit using the saturated grazing model when $p_{0}$ and $p(t)$ are both greater than $P_{\mathrm{s}}$. The dotted line shows values obtained from the best estimate model when there is a shift from linear to saturated grazing as the phytoplankton concentration exceeds $P_{\mathrm{s}}$ in the course of the incubation fore, be linearly proportional to the phytoplankton concentration as written in Eqs $(1 \& 2)$. This assumption is consistent with measurements plotted in Fig. 1 when the fraction of unfiltered seawater is small $(D<0.3)$. Assuming the time scale for ingestion of a captured phytoplankton cell is a constant, then we expect grazing will increase proportional to $p$ up until some saturation concentration $P_{\mathrm{s}}$ beyond which it remains constant. Eq. (2) can, therefore, be written as a split equation:

$$
\frac{\mathrm{d} p}{\mathrm{~d} t}= \begin{cases}\mu p-g D p & \text { if } p<P_{\mathrm{s}} \\ \mu p-g D P_{\mathrm{s}} & \text { if } p \geq P_{\mathrm{s}}\end{cases}
$$

in order to accommodate a wider range of phytoplankton concentrations.

The solution to Eq. (4) is given by Eq. (3) when $p<P_{\mathrm{s}}$. If grazing is saturated $\left(p \geq P_{\mathrm{s}}\right)$, the solution to Eq. (4) can be rearranged into the following expression for apparent growth rate:

$$
\frac{1}{t} \ln \frac{p(t)}{p_{0}}=\frac{1}{t} \ln \left\{\exp (\mu t)+\frac{g P_{\mathrm{s}}}{\mu P_{0}}[1-\exp (\mu t)]\right\}
$$

where we have used the initial condition $p_{0}=P_{0} D$. The solution represented by Eq. (5) is obtained with the assumption that grazing rate $g$ (and hence zooplankton concentration $Z$ ) does not change during the incubation. Appendix A demonstrates the solution when this assumption is not made and illustrates how the dilution method can still be useful when $Z$ changes during incubation.

The apparent phytoplankton growth rate $(\mu)$ in Eq. (5) is independent of dilution level $(D)$ when grazing is saturated. If a dilution experiment shows that growth rate is independent of $D$ in the slightly diluted treatments, then a saturated grazing model is applicable. The measurements plotted in Fig. 1 show no statistically meaningful tendency for apparent growth rate to systematically vary with $D$ when $D \geq 0.5$. In such circumstances we can calculate the phytoplankton concentration at which grazers become saturated $\left(P_{\mathrm{s}}\right)$ and the saturated grazing $\left(G_{\mathrm{s}}=g P_{\mathrm{s}}\right)$ as follows.

First rearrange Eq. (5) and make the substitution $P_{0}=p_{0} / D$ in order to obtain the following expression for $P_{\mathrm{s}}$ :

$$
P_{\mathrm{s}}=\frac{\mu\left[p(t)-p_{0} \exp (\mu t)\right]}{g D[1-\exp (\mu t)]}
$$

The variables $\mu$ and $g$ have already been obtained from more dilute samples where Eq. (3) applies. Incubating a single sample, with $p_{0} \geq P_{\mathrm{s}}$ and substituting values of $p(t), p_{0}, t$, and $D$ into the right hand side of Eq. (6) gives a single estimate of $P_{\mathrm{s}}$. Note, Eq. (6) solves for a single variable $P_{\mathrm{s}}$ so only one saturated sample needs to be incubated, although many samples should be incubated in order to confirm that grazing is truly 


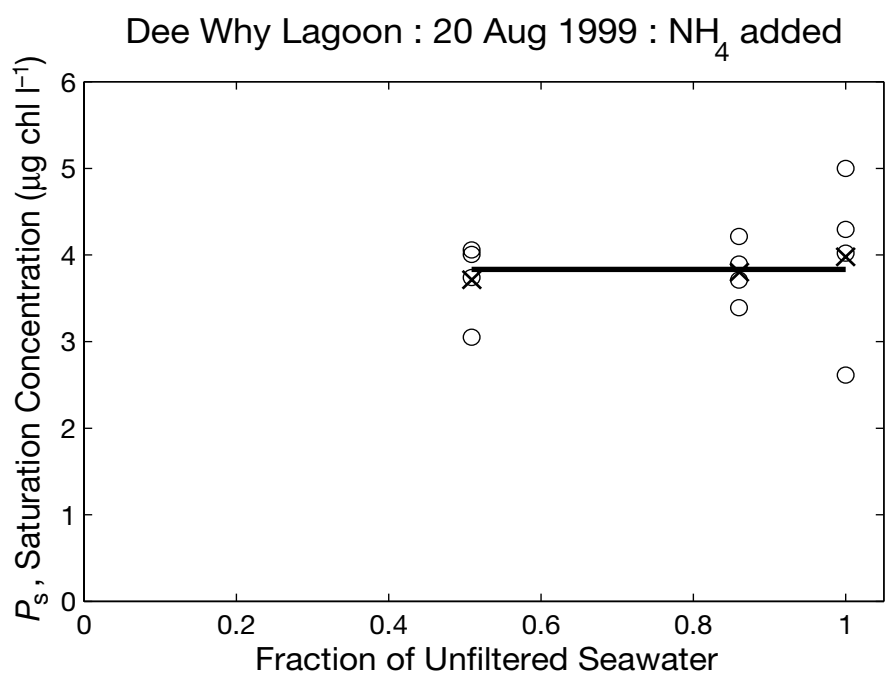

Fig. 2. Phytoplankton concentration at which grazing becomes saturated $P_{\mathrm{s}}$, plotted against fraction unfiltered seawater $D=p_{0} / P_{0}$. Circles represent values obtained from each measurement. Crosses show average values of $P_{\mathrm{s}}$ at each value of $D$. The horizontal line shows the value of $P_{\mathrm{s}}$ averaged over all measurements with $D \geq 0.5$

saturated. Also, by incubating many saturated samples, a mean value and standard error for $P_{\mathrm{s}}$ can be obtained.

Open circles in Fig. 2 show values of $P_{\mathrm{s}}$ calculated independently from each experimental determination of the phytoplankton growth in incubations where $D \geq 0.5$. Averaging $P_{\mathrm{s}}$ at each value of $D$ gives the crosses which show no obvious dependence on $D$ and which are all very close to the value averaged over all incubations with $D \geq 0.5$ (plotted with a horizontal line). The fact that the crosses fall so close to the horizontal line supports the use of the split-function model Eq. (4) as opposed to some other nonlinear function.

The development of the above experimental methodology for using Eq. (6) to determine $P_{\mathrm{s}}$ explicitly requires that $p \geq P_{\mathrm{s}}$ throughout the incubation period of the saturated sample. Determination of $g$ and $\mu$ from Eq. (3) also explicitly requires that $p<P_{\mathrm{s}}$ throughout the incubation of these more diluted samples. Table 1 presents initial and final concentrations from the less diluted incubations plotted in Figs 1 \& 2 . An estimate of $P_{\mathrm{s}}$ is obtained for each of these samples from which an overall mean value $( \pm \mathrm{SE})$ of $P_{\mathrm{s}}=3.83 \pm 0.18 \mu \mathrm{g} \mathrm{chl} \mathrm{l}^{-1}$ is obtained.

The split Eq. (4) is a simple model that involves a small number of assumptions and the empirical constants $\mu, g$ and $P_{\mathrm{s}}$ which have been measured above. It is instructive to visualize how well Eq. (4) actually fits the experimental data.

Obtaining $p(t)$ using Eq. (4) with the empirically measured constants $\mu=0.53 \mathrm{~d}^{-1}, g=0.65 \mathrm{~d}^{-1}, P_{\mathrm{s}}=3.8$ $\mu \mathrm{g} \mathrm{chl} \mathrm{l}^{-1}$ and an incubation period $t=1 \mathrm{~d}$ gives the 2 solid lines fitted through the experimentally obtained points in Fig. 1. There are no obvious nonlinear trends in the data that are not present in the split model.

It is not correct to extend the 2 lines of best fit in Fig. 1 to a point of intersection. The model fit in Fig. 1 is not, therefore, plotted for dilutions where $p_{0}<P_{\mathrm{s}}$ and $p(t)>P_{\mathrm{s}}$. Measurements were not made for dilutions corresponding to $p_{0}<P_{\mathrm{s}}$ and $p(t)>P_{\mathrm{s}}$. One might expect, however, that for dilutions corresponding to $p_{0}<P_{\mathrm{s}}$ and $p(t)>P_{\mathrm{s}}$, the experimental results would depart from extensions of the 2 linear lines because samples will have grown initially according to Eq. (3) and then according to Eq. (5), as indicated by the dotted line in Fig. 1. If additional data were available in the domain spanned by the dotted line, then a nonlinear regression would be required in order to include the additional data in the calculation of $\mu, g$ and $P_{\mathrm{s}}$.

We also note that the assemblage of grazers is highly diverse and that $P_{\mathrm{s}}$ represents a community average. The grazing by different zooplankton species within the community would likely saturate at different phytoplankton concentrations, so we expect there should be some curvature introduced near the point of intersection obtained by extending the two linear lines in Fig. 1.

It is notable that $g>\mu$ in the single experiment analyzed here. If we take these values of $g$ and $\mu$ to be fixed, then microzooplankton grazing controls the phytoplankton abundance by causing it to decay exponentially at a rate $g-\mu$ when $P \leq P_{\mathrm{s}}$. Phytoplankton concentrations in the range $P_{\mathrm{s}}<P<g P_{\mathrm{s}} / \mu$ will also be grazed away, but at a lesser rate.

The maximum rate at which microzooplankton consumed phytoplankton in the Dee Why Lagoon experiment is given by the saturated grazing $G_{\mathrm{s}}=g P_{\mathrm{s}}$ which

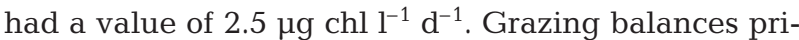
mary production when the phytoplankton concentration is $P_{\mathrm{b}}=g P_{\mathrm{s}} / \mu$. The value for this balanced phytoplankton concentration was $P_{\mathrm{b}}=4.76 \mu \mathrm{g}$ chl $\mathrm{l}^{-1}$ which

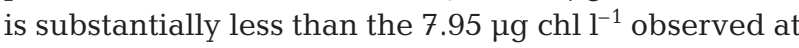
the study site. In the example measured, microzooplankton grazing is not sufficient to totally control phytoplankton growth.

Table 1. Initial $\left(p_{0}\right)$ and final $[p(t)]$ phytoplankton concentrations ( $\mu \mathrm{g} \mathrm{chl} \mathrm{l}^{-1}$ ) and the average value of the phytoplankton concentration that saturates grazing $\left(P_{\mathrm{s}}\right)$ at 3 values of $D$ (fraction unfiltered seawater) for a dilution experiment conducted in Dee Why Lagoon, August 1999

\begin{tabular}{|cccc|}
\hline$D$ & $\begin{array}{c}p_{0} \\
\mu g \text { chl l}^{-1}\end{array}$ & $\begin{array}{c}p(t) \\
\mu g \text { chl l}^{-1}\end{array}$ & $\begin{array}{c}\text { Average } P_{\mathrm{s}} \\
\mu g \text { chl l}^{-1}\end{array}$ \\
\hline 1 & 7.95 & $9.16,10.0,9.77,11.2$ & 3.98 \\
0.86 & 6.84 & $9.07,8.84,8.47,8.70$ & 3.80 \\
0.51 & 4.05 & $5.07,5.21,5.10,5.52$ & 3.71 \\
\hline
\end{tabular}


On the basis of our single experiment it would seem that an algal bloom might be expected in Dee Why Lagoon unless something other than water-column grazing by microzooplankton is limiting the phytoplankton concentration. Nutrient limitation in Dee Why Lagoon was unlikely, because phytoplankton growth in dilution samples that were spiked with nutrients was similar to growth in replicate samples that did not receive additional nutrients. It should also be noted that Dee Why Lagoon is situated in a highly urbanized area and receives significant storm water inputs. It is possible that larger water-column grazers, such as copepods, might modify $P_{\mathrm{b}}$ to be larger than the initial phytoplankton concentration, $7.95 \mu \mathrm{g} \mathrm{chl} \mathrm{l}^{-1}$. The other obvious mitigating candidate is light. Incubations for the dilution experiment were carried out at a fixed depth on a sunny day. Reduced light levels would be expected to reduce $\mu$ (but not $g$ or $P_{\mathrm{s}}$ ), in which case $P_{\mathrm{b}}$

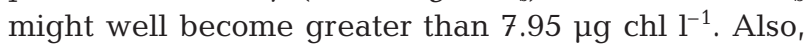
hyper-benthic grazers may help control phytoplankton concentrations, given that nighttime cooling of shallow lagoons often results in convection throughout the depth of the water column. Finally, there is always the possibility that microzooplankton abundance might increase and thereby increase grazing impact. Clearly, the dilution method does not measure all the processes controlling phytoplankton concentrations in natural systems. Rather, the dilution method yields quantitative information about parameters that are necessary but not sufficient to determine the extent of microzooplankton grazing on the phytoplankton.

The piecewise linear model has been applied here to the interpretation of a single set of measurements. It would not be unreasonable, however, to interpret the Gallegos (1989) and Dolan et al. (2000) experiments in the context of the piecewise linear model, although other less tractable nonlinear models might equally apply within the measurement certainty. In the absence of measurements requiring a more sophisticated analysis, we suggest that the extension to the dilution method presented here is useful for interpreting dilution experiments conducted in productive waters.

It may be that future dilution experiments are made with a precision sufficient to reject our piecewise linear model in favor of a grazing model that is relatively difficult (or impossible) to solve analytically. In such circumstances we expect that the piecewise interpretation would still serve as an accessible tool that gives an answer to within a usable approximation.

\section{CONCLUSIONS}

A straightforward split-function model for zooplankton grazing has been used to extend the applicability of the dilution method to phytoplankton concentrations at which microzooplankton grazing becomes saturated. The model fits the observations well within the margins of measurement error and has the advantage of being analytically tractable. Exponential phytoplankton growth and microzooplankton grazing rates are determined from sufficiently diluted samples in the conventional manner by using the linear relation between apparent growth rate and dilution. The extension provided here enables estimation of the phytoplankton concentration $P_{\mathrm{s}}$ at which the rate of consumption by zooplankton becomes saturated. All assumptions are tacit within the choice of the split-function model, and no assumptions are introduced in the mathematical technique by which the equations are used to analyze experimental results. This is in contrast with Gallegos' (1989) considerations, which assume nonlinear models and make further assumptions in the analytic techniques by which these models are fitted to experimental results.

The dilution method can be used to obtain 3 independent parameters, namely growth rate $(\mu)$, grazing rate $(g)$ and the concentration at which grazers become saturated $\left(P_{\mathrm{s}}\right)$. These parameters and the calculated value for saturated grazing $\left(G_{\mathrm{s}}=g P_{\mathrm{s}}\right)$ are necessary to estimate phytoplankton growth and the impact of microzooplankton grazers in mesotrophic and eutrophic systems. As such, the dilution method provides information fundamental for understanding processes in waters that are subject to high primary productivity as a result of substantial nutrient loading.

Acknowledgements. Funding from the NSW Department of Land and Water Conservation supported the field component of this work. The authors are grateful to 4 anonymous reviewers who provided helpful suggestions for improving this manuscript.

\section{LITERATURE CITED}

Dolan JR, Gallegos CL, Moigis A (2000) Dilution effects on microzooplankton in dilution grazing experiments. Mar Ecol Prog Ser 200:127-139

Evans GT, Paranjape MA (1992) Precision of estimates of phytoplankton growth and microzooplankton grazing when the functional response of grazers may be nonlinear. Mar Ecol Prog Ser 80:285-290

Gallegos CL (1989) Microzooplankton grazing on phytoplankton in the Rhode River, Maryland: nonlinear feeding kinetics. Mar Ecol Prog Ser 57:23-33

Gallegos CL, Vant WN, Safi KA (1996) Microzooplankton grazing of phytoplankton in Manukau Harbour, New Zealand. NZ J Mar Freshw Res 30:423-434

Gifford DJ, Fessenden LM, Garrahan PR, Martin E (1995) Grazing by microzooplankton and mesozooplankton in the high-latitude North Atlantic Ocean: spring versus summer dynamics. J Geophys Res 100:6665-6675

Holling CS (1959) Some characteristics of simple types of predation and parasitism. Can Entomol 91:385-398 
Landry MR (1994) Methods and controls for measuring the grazing impact of planktonic protists. Mar Microb Food Webs 8:37-57

Landry MR, Hassett RP (1982) Estimating the grazing impact of marine microzooplankton. Mar Biol 67:283-288

Landry MR, Kirshtein J, Constantinou J (1995) A refined dilu-

Editorial responsibility: Otto Kinne (Editor),

Oldendorf/Luhe, Germany tion technique for measuring the community grazing impact of microzooplankton, with experimental tests in the central equatorial Pacific. Mar Ecol Prog Ser 120:53-63

Putland JN (2000) Microzooplankton herbivory and bacterivory in Newfoundland coastal waters during spring, summer and winter. J Plankton Res 22:253-277

Submitted: December 12, 2000, Accepted: May 8, 2001 Proofs received from author(s): January 11, 2002
APPENDIX A. The effect of zooplankton growth on the piecewise linear dilution method will now be analyzed. Instead of Eq. (4) we would have the following coupled equations

$$
\begin{aligned}
& \frac{\mathrm{d} p}{\mathrm{~d} t}= \begin{cases}\mu p-\gamma z p & \text { if } p<P_{\mathrm{s}} \\
\mu p-\gamma z P_{\mathrm{s}} & \text { if } p \geq P_{\mathrm{s}}\end{cases} \\
& \frac{\mathrm{d} z}{\mathrm{~d} t}= \begin{cases}\alpha \gamma z p & \text { if } p<P_{\mathrm{s}} \\
\alpha \gamma z P_{\mathrm{s}} & \text { if } p \geq P_{\mathrm{s}}\end{cases}
\end{aligned}
$$

where $z=D Z$ is the diluted zooplankton concentration and $g=\gamma Z$. Note, the equation for zooplankton growth makes the assumption that the microzooplankton feed only on the phytoplankton and grow with efficiency $\alpha$.

As the sample is made more dilute it is clear that the phytoplankton growth rate, $(1 / p) \mathrm{d} p / \mathrm{d} t$, tends to $\mu$ whereas that of the zooplankton, $(1 / z) \mathrm{d} z / \mathrm{d} t$, tends to 0 . On this basis, issues relating to zooplankton growth appear to become unimportant at high dilutions, but this is not entirely so. Dolan et al. (2000) observed that sufficiently high dilutions result in the mortality of some zooplankton groups. Eq. (A-2) makes no allowance for mortality when $p \ll P_{\mathrm{s}}$. Regardless, the phytoplankton growth rate $\mu$ is still the $y$-intercept on the dilution plot although the approach to this intercept will flatten at small dilutions. Given the experimental scatter and small number of different dilutions typically used in dilution experiments, it would be difficult (but in principle possible) to unambiguously determine the modification of grazing due to differential mortality and growth of zooplankton at different dilutions from the standard protocol used in dilution experiments. Dolan et al. (2000) extended the usual protocol by measuring various groups of grazers and demonstrated that different grazers have quite different response functions to changes in phytoplankton concentration (Dolan et al. 2000).

Zooplankton mortality is probably not an issue when the concentrations are high enough for satu- rated grazing. It is easy to integrate Eq. (A-2) to obtain

$$
\mathrm{z}=\mathrm{z}_{0} \exp \left(\alpha \gamma P_{\mathrm{s}} t\right)
$$

which upon substitution into the equation for phytoplankton growth gives

$$
\frac{\mathrm{d} p}{\mathrm{~d} t}=\mu p-\gamma P_{\mathrm{s}} z_{0} \exp \left(\alpha \gamma P_{\mathrm{s}} t\right)
$$

where the $z_{0}=D Z_{0}$, with $Z_{0}$ being the microzooplankton of the field sample at the time it was collected. The solution to Eq. (A-4), subject to the initial phytoplankton concentration $p_{0}$, can be written in terms of apparent growth

$\frac{1}{t} \ln \frac{p(t)}{p_{0}}=$

$\frac{1}{t} \ln \left\{\exp (\mu t)+\frac{\gamma Z_{0} P_{\mathrm{s}}}{P_{0}\left(\alpha \gamma P_{\mathrm{s}}-\mu\right)}\left[\exp (\mu t)-\exp \left(\alpha \gamma P_{\mathrm{s}} t\right)\right]\right\}$

where the substitutions $p_{0}=P_{0} D$ and $\gamma z_{0}=\gamma Z_{0} D$ have been used. It is clear that Eq. (A-5) reduces to Eq. (5) when $\alpha \gamma P_{\mathrm{s}} \ll \mu$.

In the instance when $\alpha \gamma P_{\mathrm{s}}=\mu$ (microzooplankton and phytoplankton growing at the same exponential rate), the appropriate form of the above equation for the apparent growth rate is

$$
\frac{1}{t} \ln \frac{p(t)}{p_{0}}=\frac{1}{t} \ln \left\{\exp (\mu t)+\frac{\gamma Z_{0} P_{\mathrm{s}}}{P_{0}} t \exp (\mu t)\right\}
$$

Regardless of growth of microzooplankton, it is clear (from the 2 preceding equations) that the apparent growth rate is still independent of the fraction of unfiltered seawater $D$ when the grazing of microzooplankters is saturated.

It seems, in principle, possible to obtain $\mu, \gamma, P_{\mathrm{s}}$ and $\alpha$ from a sufficiently precise dilution experiment in which $Z_{0}$ is also determined. Whether or not this can be done in practice remains to be demonstrated. 